\title{
Trans-sternotomy versus video-assisted thoracic surgery for early-stage thymoma patients: a meta-analysis
}

\author{
Aizemaiti Rusidanmu ${ }^{1}$, Mingyang Feng ${ }^{2}$, Jinming Xu ${ }^{1}$, Luming Wang ${ }^{1}$, Cheng $\mathrm{He}^{1}$, Jian Hu ${ }^{1}$ \\ ${ }^{1}$ Department of Thoracic Surgery, the First Affiliated Hospital, Zhejiang University School of Medicine, Hangzhou 310003, China; ${ }^{2}$ Zhejiang \\ university school of Medicine, Hangzhou 310029, China \\ Contributions: (I) Conception and design: J Hu, A Rusidanmu; (II) Administrative support: None; (III) Provision of study materials or patients: None; \\ (IV) Collection and assembly of data: M Feng, J Xu; (V) Data analysis and interpretation: M Feng, J Xu; (VI) Manuscript writing: All authors; (VII) \\ Final approval of manuscript: All authors. \\ Correspondence to: Prof. Jian Hu, MD, PhD. Department of Thoracic Surgery, The first Affiliated Hospital, Zhejiang University School of Medicine, \\ Hangzhou 310003, China. Email: dr_hujian@zju.edu.cn.
}

Background: Over the years, video-assisted thoracic surgery (VATS) thymectomy has progressively replaced trans-sternotomy (TS) in early-stage thymoma (Masaoka stage I and stage II). This meta-analysis aimed to confirm the differences in the efficacies of VATS and TS approaches in early-stage thymoma patients.

Methods: A thorough literature search of the following online databases was performed: PubMed, Cochrane Library, Web of Science, and EMBASE. Appropriate search terms, such as "thymoma or thymus neoplasms or Thymic Carcinoma" and "Video-Assisted Thoracic Surgeries or Video-Assisted Thoracoscopic", were used with MeSH search methods. Heterogeneity was assessed first with the Q-test and inconsistency index and sensitivity analysis and subgroup analysis were then used to find the source of heterogeneity.

Results: We retrieved 1,228 articles, 11 articles were selected as the subjects of our research, and 1,222 patients were included in the research (666 VATS cases versus 556 TS cases). VATS caused less blood loss $(\mathrm{P}=0.02)$, and required shorter hospital stay $(\mathrm{P}<0.001)$, shorter duration of chest tube drainage $(\mathrm{P}=0.03)$ than TS. No obvious difference was found in operative time $(\mathrm{P}=0.14)$, postoperative recurrence $(\mathrm{OR}=0.81$, 95\% CI: 0.35-1.85, P=0.613), postoperative complications ( $\mathrm{OR}=0.60,95 \% \mathrm{CI}: 0.31-1.16, \mathrm{P}=0.129)$ and $\mathrm{R} 0$ resection $(\mathrm{OR}=0.35,95 \% \mathrm{CI}: 0.12-1.04, \mathrm{P}=0.06)$, but the trend showed that more patients in the TS group achieved R0 resection.

Conclusions: For early-stage thymoma patients, VATS thymectomy seems to provide many advantages to be considered as a legitimate alternative to TS; however, when performing VATS, surgeons should pay special attention to ensure that $\mathrm{R} 0$ resection is achieved.

Keywords: Meta-analysis; thymoma, thymectomy; video-assisted thoracic surgery (VATS)

Submitted Nov 16, 2019. Accepted for publication Feb 14, 2020.

doi: 10.21037 /gs.2020.03.10

View this article at: http://dx.doi.org/10.21037/gs.2020.03.10

\section{Introduction}

The surgical resection of an early-stage thymoma (Masaoka stage I and stage II) via trans-sternotomy (TS) is the standard treatment approach, as endorsed by major guidelines. This approach provides a massive exposure of the anterior mediastinum, while allowing an extended thymectomy with the exenteration of the whole mediastinal fat, which are the two mainstays of the therapeutic success of the procedure. Unfortunately, TS causes significant tissue trauma, with morbidity and postoperative complications, leading to extended hospital stay. It also leaves an unpleasant 
scar and thus has a very low acceptance rate in the patient population, which is mainly represented by young women (1).

Video-assisted thoracic surgery (VATS) for thymectomy, including bilateral and unilateral VATS (either left or right) approaches, has evolved significantly over the last decade. VATS offers superior illumination and magnification, and with the availability of advanced cameras with variable angles, it also provides better exposure and lighting of the operative field (2). Although VATS is not routinely recommended because its long-term data are still lacking (3), increasing numbers of studies have reported that VATS is a safe and effective procedure for treating early-stage thymoma and exhibits satisfactory prognosis (4). This meta-analysis aimed to confirm the differences in the efficacies of VATS and TS approaches in early-stage thymoma patients and was designed based on the PRISMA guidelines (5).

\section{Methods}

A thorough literature search of the following online databases was performed: PubMed, Cochrane Library, Web of Science, and EMBASE. Appropriate search terms, such as "thymoma or thymus neoplasms or Thymic Carcinoma" and "Video-Assisted Thoracic Surgeries or Video-Assisted Thoracoscopic", were used with MeSH search methods.

\section{Study selection and inclusion criteria}

Studies were included in the meta-analysis if they met the following criteria: (I) they were randomized controlled trials (RCTs) or cohort studies; (II) their subjects were early-stage thymoma patients (Masaoka stage I or II) who underwent thymectomy; (III) they compared VATS and TS outcomes; (IV) outcome indicators included blood loss, operative time, length of hospital stay, duration of chest tube drainage, complications, recurrence, etc.; (V) the articles were published in English.

\section{Exclusion criteria}

Studies were excluded from the meta-analysis in the following cases: (I) they were case reports, reviews, or conference abstracts of which full texts were not available; (II) the procedures involved robotic-assisted thoracic surgeries (RATS) or trans-cervical surgery; (III) they were for other benign conditions alone (e.g., myasthenia gravis and thymolipoma) or non-thymic malignancies alone (e.g., germ cell tumors, lymphoma, and lung cancer) or were one armed studies; (IV) they were published before January 2010 .

\section{Data extraction}

Two investigators independently conducted the preliminary screening by reading titles and abstracts under the guidance of faculty members from the same center. Information extracted from the articles included the first author's name, publication year, country or district where the study was conducted, the number of patients in each group, and surgical procedures. The full texts of papers that met the inclusion criteria were then reviewed. Study end points included some or all of the following: mean blood loss (milliliters), operative time (minutes), length of hospital stay (days), duration of chest tube drainage (days), postoperative complication rate, resection margin, and recurrence rate.

In practice, many authors had used propensity scores for balancing age, gender, and mean tumor size of the VATS and TS groups in their studies, so comparisons between these end points were avoided.

\section{Statistical analysis}

To assess the quality of the included studies, the Methodological Index for Non-Randomized Studies (MINORS) (6) was applied to all of the included studies.

Stata/SE 14.0 (StataCorp, College Station, USA) was used to estimate statistical significance. Heterogeneity was assessed first using the Q-test and inconsistency index. A random effects model was applied to every comparison. The odds ratios (ORs) were calculated for binary outcomes and the standardized mean differences for continuous outcomes. All $\mathrm{P}$ values were two-sided, and a $\mathrm{P}$ value of $<0.05$ was considered statistically significant.

\section{Results}

\section{Results of filtering literature}

We retrieved 1,228 relevant articles through the database searches: 427 from EMBASE, 114 from Cochrane Library, 379 from Web of Science, and 308 from PubMed. Of these articles, 1,124 were excluded for the following reasons: 514 were duplicates, 79 were case reports, 46 included DaVinci RATS, 5 were meta-analyses, and the other 480 were 


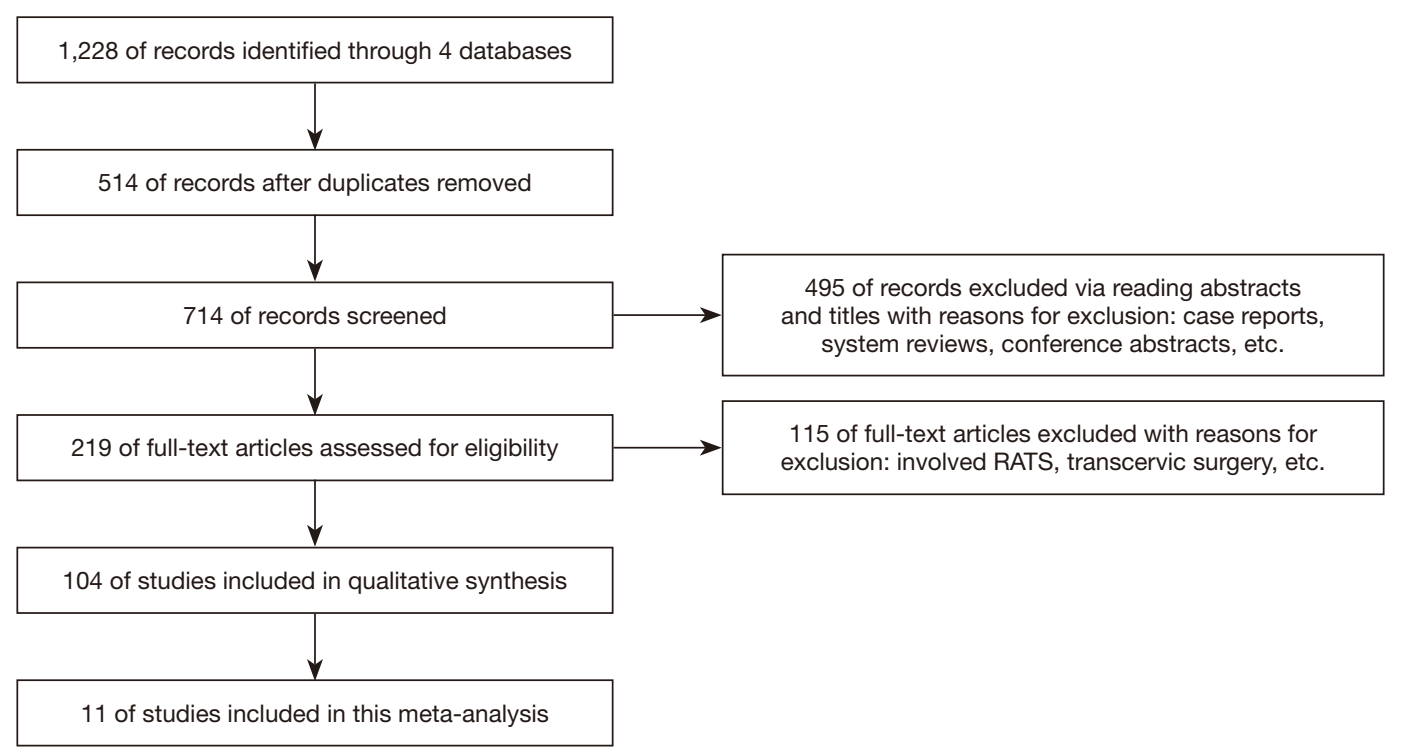

Figure 1 The process of selecting eligible articles for further research.

systematic reviews, conference abstracts, or unpublished prospective studies. The abstracts of the remaining 104 articles were examined for the inclusion and exclusion criteria. This process led to a further exclusion of 70 articles. The full text of the remaining 34 articles was then reviewed. Finally, 11 articles were included in our analysis (Figure 1).

\section{Characteristics of the included studies}

All of the included 11 articles, with publication dates ranging from 2010 to 2018, were based on nonrandomized and retrospective studies as no standardized RCT on this topic has been published yet. Basic information of 11 cohorts from these studies, giving a total of 1,222 cases (666 VATS cases and 556 TS cases), was included in the analysis. Detailed information of each study is provided in Table 1. The MINORS criteria (Table 2) were applied to all 11 studies (mean score, 18.7).

\section{Description of surgical procedures}

Unilateral VATS was the most common procedure among the included studies $(n=9)$. In six of these studies, the leftor right-side approach was used based on the position of the thymoma. Only two studies used bilateral VATS $(14,17)$. The detailed surgical procedures of TS were similar between all of the included trials.

\section{Surgical parameters}

The mean blood loss and the mean operative time were important parameters for comparing VATS and TS. The heterogeneity was significant in both parameters (inconsistency index $=96.1 \%$ and $89.3 \%$, respectively). Compared with TS, VATS caused less blood loss $(\mathrm{P}=0.02)$ (Figure $2 A)$ and required equal operative time $(\mathrm{P}=0.14)$ (Figure $2 B$ ). Subgroup analysis by country was used to reduce the heterogeneity in operative time.

\section{Post-surgical parameters}

The length of hospital stay and the duration of chest tube drainage were the post-surgical parameters evaluated in this meta-analysis. The heterogeneity was significant in both parameters (inconsistency index $=83.2 \%$ and $95.2 \%$, respectively). Compared with TS patients, VATS patients had a shorter hospital stay $(\mathrm{P}<0.001)($ Figure $3 A)$ and a shorter duration of chest tube drainage $(\mathrm{P}=0.03)$ (Figure $3 B)$.

\section{Postoperative complications}

The heterogeneity in postoperative complications was not significant (inconsistency index $=16.8 \%$ ). The forest plot (Figure 4) showed no statistically significant difference in the incidence of postoperative complications between the VATS and TS groups $[\mathrm{OR}=0.60,95 \%$ confidence interval (CI): 0.31-1.16, $\mathrm{P}=0.129]$, although the trend depicted 
Table 1 Information on the 11 trails included in the meta-analysis

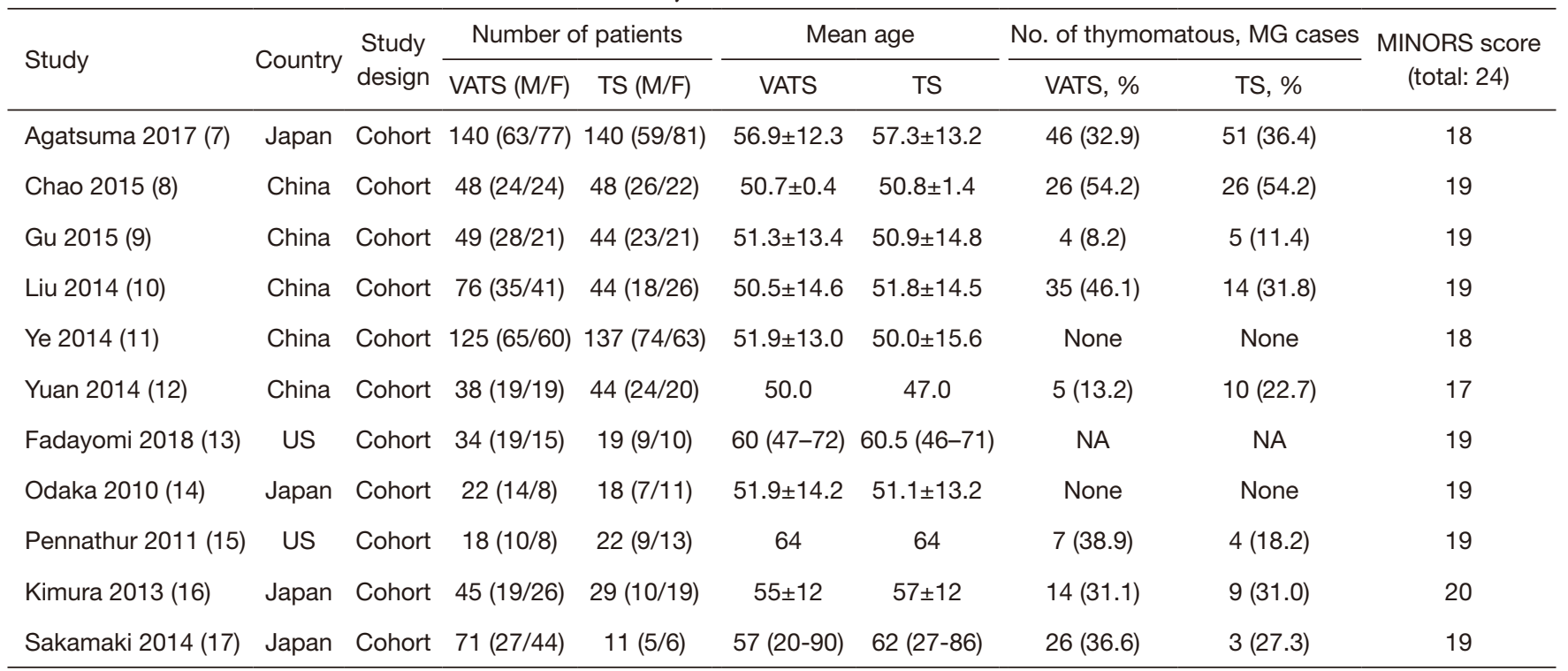

NA, Not available; M, male; F, female; VATS, video-assisted thoracic surgery; TS, trans-sternotomy; MG, myasthenia gravis; MINORS, methodological index for non-randomized studies.

Table 2 The MINORS score of each study

\begin{tabular}{|c|c|c|c|c|c|c|c|c|c|c|c|c|c|}
\hline Study & \multicolumn{12}{|c|}{ Methodological index for non-randomized studies } & Tota \\
\hline Agatsuma 2017 & 2 & 2 & 2 & 2 & 0 & 2 & 2 & 0 & 2 & 2 & 1 & 1 & 18 \\
\hline Chao 2015 & 2 & 2 & 2 & 2 & 0 & 2 & 2 & 0 & 2 & 2 & 1 & 2 & 19 \\
\hline Gu 2015 & 2 & 2 & 2 & 2 & 0 & 1 & 2 & 0 & 2 & 2 & 2 & 2 & 19 \\
\hline Ye 2014 & 2 & 1 & 2 & 2 & 0 & 1 & 2 & 0 & 2 & 2 & 2 & 2 & 18 \\
\hline Yuan 2014 & 2 & 1 & 2 & 2 & 0 & 1 & 2 & 0 & 2 & 2 & 2 & 1 & 17 \\
\hline Fadayomi 2018 & 2 & 2 & 2 & 2 & 0 & 2 & 2 & 0 & 2 & 2 & 2 & 1 & 19 \\
\hline Odaka 2010 & 2 & 2 & 2 & 2 & 0 & 1 & 2 & 0 & 2 & 2 & 2 & 2 & 19 \\
\hline Sakamaki 2014 & 2 & 2 & 2 & 2 & 0 & 1 & 2 & 0 & 2 & 2 & 2 & 2 & 19 \\
\hline
\end{tabular}

1, A stated aim of the study; 2, inclusion of consecutive patients; 3 , prospective collection of data; 4 , endpoint appropriate to the study aim; 5 , unbiased evaluation of endpoints; 6 , follow-up period appropriate to the major endpoint; 7 , loss to follow up not exceeding $5 \%$; 8 , a control group having the gold standard intervention; 9, Contemporary groups; 10, baseline equivalence of groups; 11, prospective calculation of the sample size; 12, statistical analyses adapted to the study design. MINORS, methodological index for non-randomized studies. 


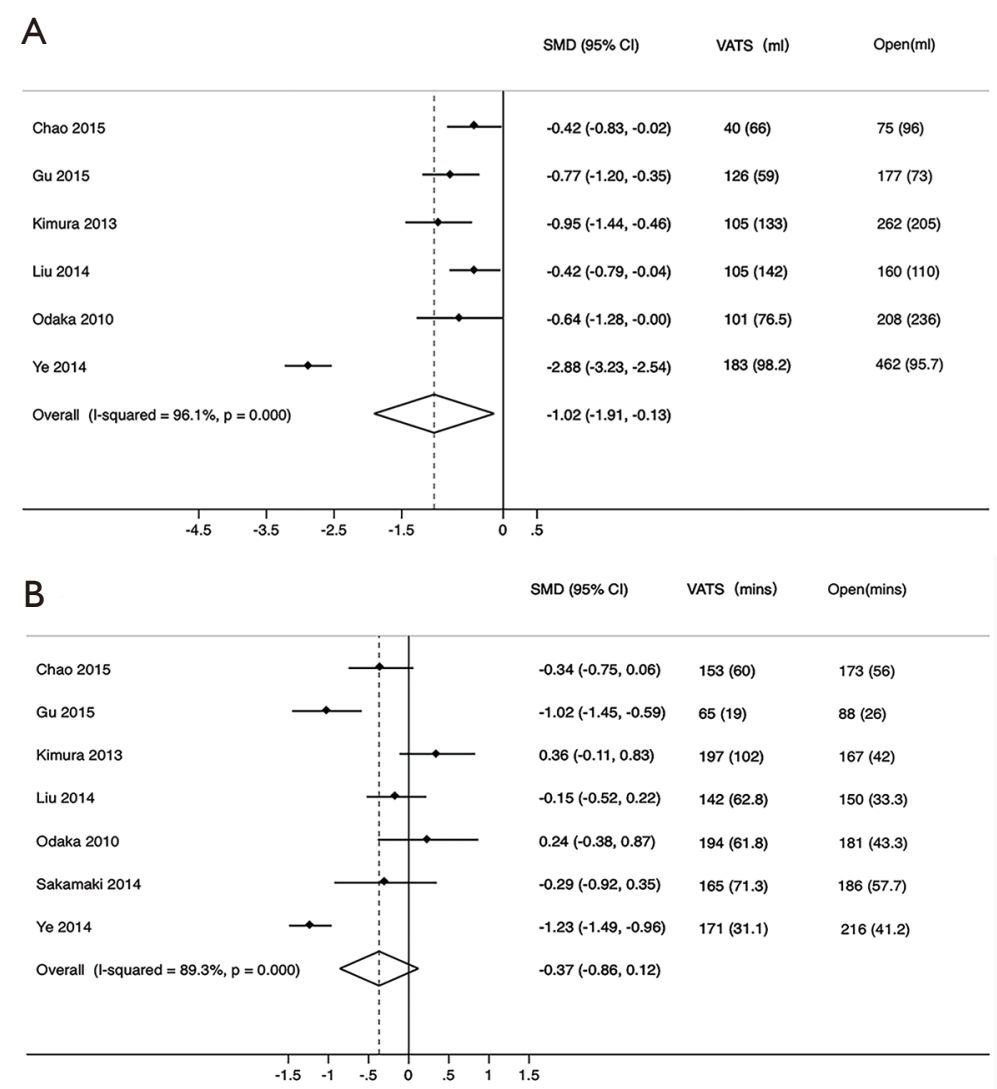

Figure 2 Surgical parameters in VATS versus TS. (A) Blood loss (mL); (B) operative time (minutes). VATS, video-assisted thoracic surgery; TS, trans-sternotomy.

fewer complications in the VATS group.

\section{RO resection}

With respect to $\mathrm{R} 0$ resection, the heterogeneity was not significant (inconsistency index $=0 \%$ ). The result (Figure 5) indicated no statistically significant difference in R0 resection between the TS or VATS groups (OR $=0.35,95 \%$ CI: 0.12-1.04, $\mathrm{P}=0.06$ ), but the trend showed that more patients in the TS group achieved $\mathrm{R} 0$ resection.

\section{Postoperative recurrence}

In terms of postoperative recurrence, the heterogeneity was not significant (inconsistency index $=0 \%$ ). The result (Figure 6) indicated no significant differences in postoperative recurrence between the VATS and TS groups $(\mathrm{OR}=0.81$; 95\% CI: $0.35-1.85, \mathrm{P}=0.613)$.

\section{Discussion}

The ability of the MINORS to identify the quality of noncomparative studies (poor or fair) has been proven (6). All 11 articles in our study were evaluated using the MINORS criteria, yielding a mean score of 18.7 points. Among the included studies, the lowest score was 17 points and the highest was 20 points, which showed that the quality of the included studies was relatively similar and could be combined.

Statistically significant clinical outcomes were decreased blood loss, shorter hospital stay, and shorter duration of chest tube drainage, all of which were found in the VATS groups.

The heterogeneity in operative time comparison was found to be significant (inconsistency index $=89.3 \%$ ); therefore, we performed a sensitivity analysis and found that the heterogeneity was mostly attributable to the study by Ye et al. (11), but we found no significant 


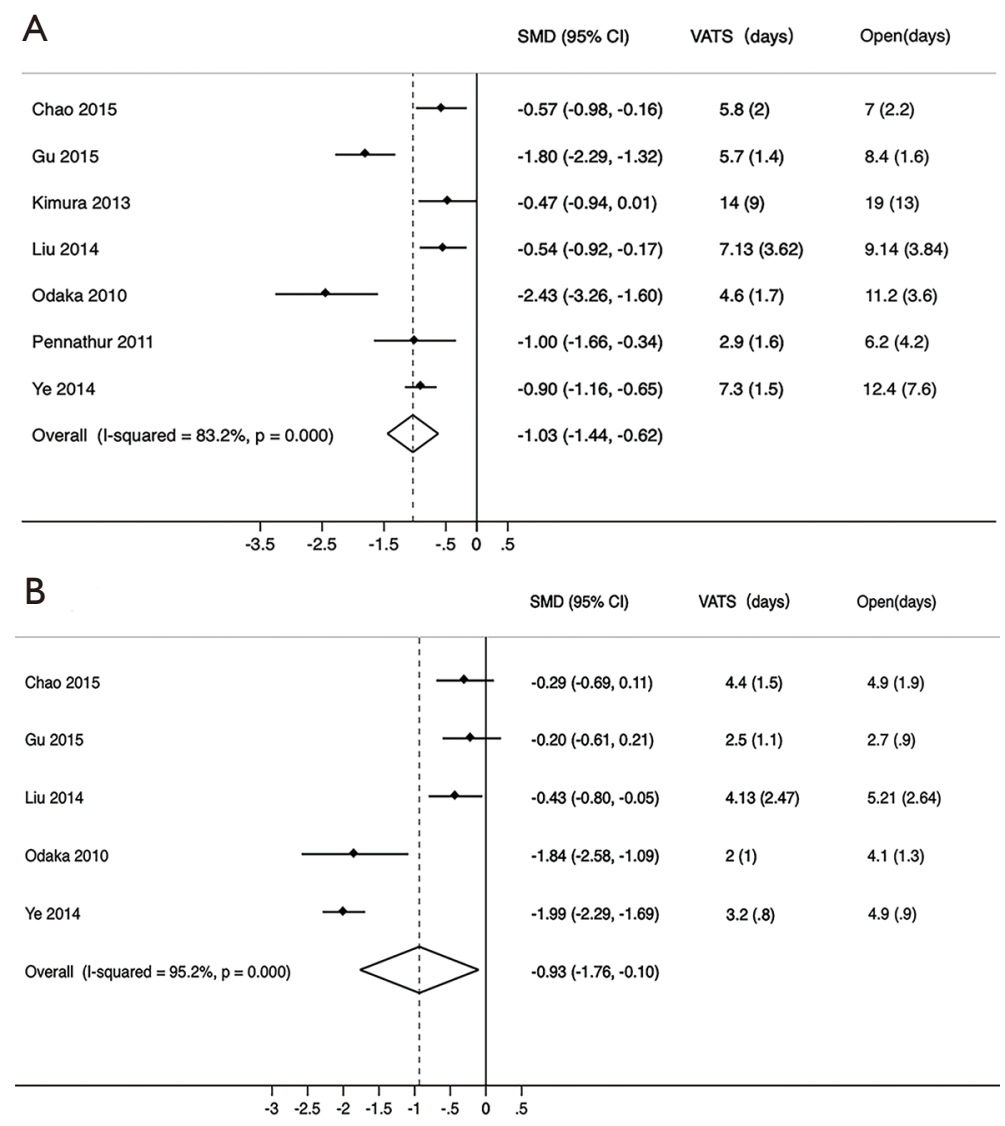

Figure 3 Post-surgical parameters in VATS versus TS. (A) Length of hospital stay; (B) duration of chest tube drainage. VATS, video-assisted thoracic surgery; TS, trans-sternotomy.

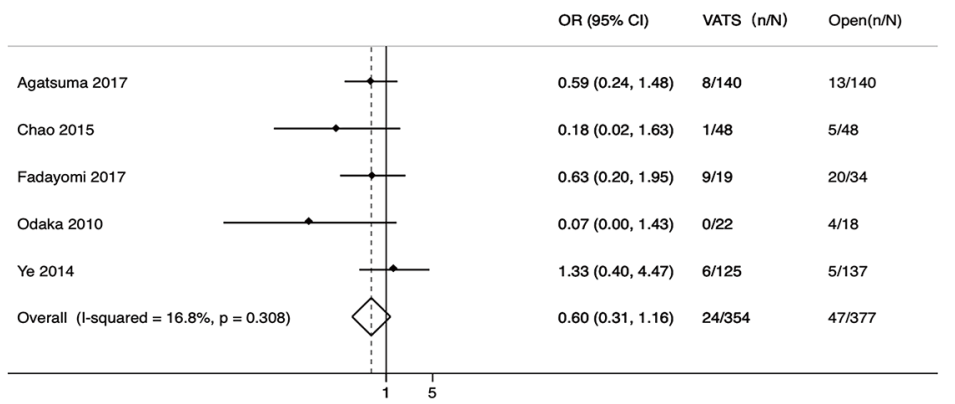

Figure 4 VATS versus TS, postoperative complication. VATS, video-assisted thoracic surgery; TS, trans-sternotomy.

difference in this study after reviewing its full text. The heterogeneity reduced a little after eliminating this study (inconsistency index $=76.6 \%$ ). Toker et al. (18) reported that operative time is correlated with the number of VATS thymectomies, which suggests that operative time differs between institutions. Moreover, although general surgical procedures and principles are similar, surgical skills tend to vary greatly between institutions. These findings may help explain the heterogeneity found in results, which could thus be considered stable. To confirm the stability of our results, we performed a subgroup analysis by country and found differences between studies from China and Japan (Figure 7). 


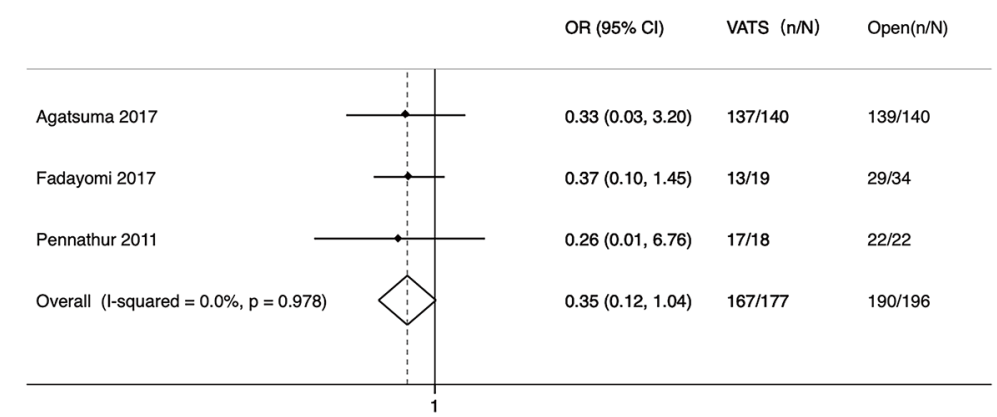

Figure 5 VATS versus TS, R0 resection. VATS, video-assisted thoracic surgery; TS, trans-sternotomy.

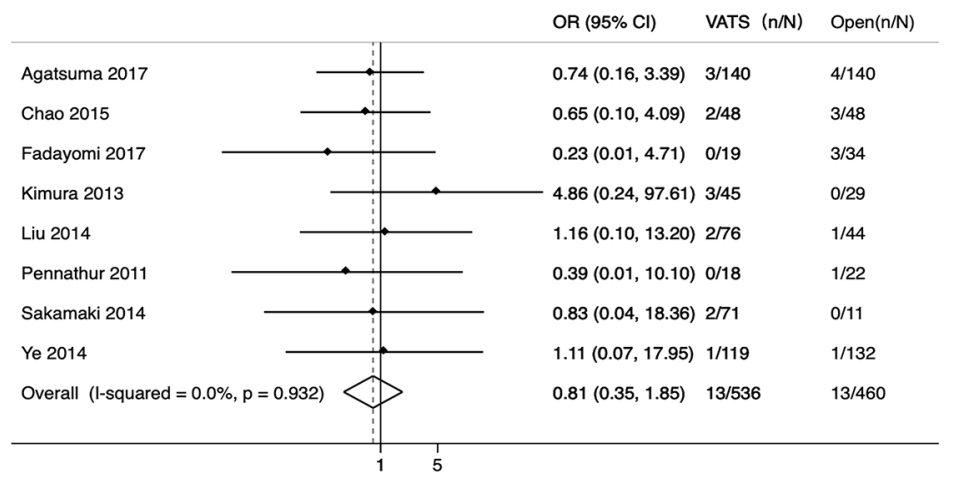

Figure 6 VATS versus TS, postoperative recurrence. VATS, video-assisted thoracic surgery; TS, trans-sternotomy.

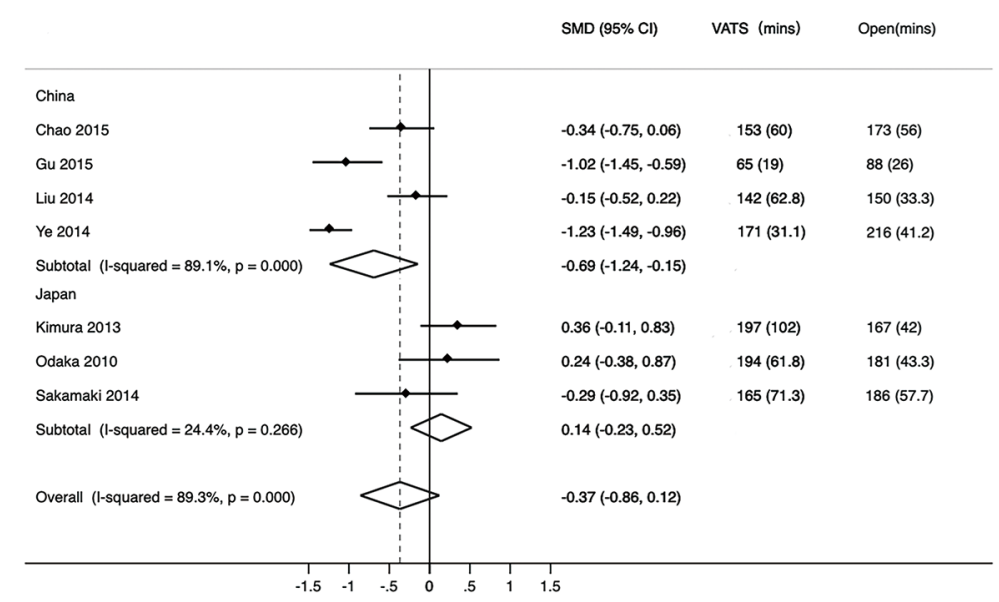

Figure 7 VATS versus TS, operative time subgroup. VATS, video-assisted thoracic surgery; TS, trans-sternotomy.

This result suggested that VATS required equal operative time to TS in Japan ( $\mathrm{P}=0.45)$ but less in China $(\mathrm{P}=0.01)$.

As for the blood loss comparison, the heterogeneity was significant (inconsistency index $=96.1 \%$ ). The sensitivity analysis showed that the studies by Liu et al. (10) and
Ye et al. (11) could be the source of heterogeneity. After eliminating both of these studies, the heterogeneity reduced (inconsistency index $=0 \%$ ). Our results suggested that the VATS approach caused less blood loss compared with TS $(\mathrm{P}<0.01)$. Notably, both eliminated studies also reported 
that VATS caused less bleeding than TS.

With regard to both the length of hospital stay and the duration of tube drainage, the heterogeneity was significant (inconsistency index $=83.2 \%$ and $95.2 \%$, respectively). The sensitivity analysis of the duration of chest tube drainage revealed that the study by Ye et al. (11) could be the source of heterogeneity. After eliminating that study, the heterogeneity reduced (inconsistency index $=80.4 \%$ ). In contrast, the sensitivity analysis of the length of hospital stay indicated no probable study that could have caused the heterogeneity. Thus, we speculated that the hospitalization indicators were different between hospitals, which may explain the heterogeneity found in our results on the length of hospital stay.

Although Lucchi et al. (19) suggested that the VATS approach increases the risk of recurrence compared with the TS approach, we observed no differences in the postoperative recurrence events between the two groups $(\mathrm{P}=0.613)$.

Further, no statistically significant difference was found in the postoperative complications between the VATS and TS groups $(\mathrm{P}=0.129)$. One study (20) reported an incidence of mediastinitis, a life-threatening complication with an associated mortality rate ranging from $10 \%$ to $47 \%$, of $1-5 \%$ after TS $(20,21)$. In view of this finding, many thoracic surgeons have turned to less invasive approaches such as VATS for early-stage thymoma. However, our results on postoperative complication showed nonsignificant differences between the TS and VATS groups, but the trend indicated fewer complications in the VATS group.

Taken together, our results suggest that VATS thymectomy for thymoma has at least equal if not superior oncological efficacy. Regarding R0 resection, although the difference was not statistically significant $(\mathrm{P}=0.059)$, the TS group showed better R0 resection outcomes than the VATS group, which is in contrast to the finding of a metaanalysis by Friedant et al. (22). According to the Cochrane Handbook chapter 16, article 9.3 (23), we excluded the studies in which all patients underwent R0 resection in both VATS and TS groups, but such studies were included in the meta-analysis by Friedant et al. (22). This may be the source of differences in the results between our study and that of Friedant et al. (22). Furthermore, we found that the inclusion/exclusion of "studies with no events" in a meta-analysis is still a controversial issue. Finally, we chose to follow the Cochrane Handbook and exclude studies with no events. However, given our small sample size and retrospective data, it cannot be concluded that VATS is inferior to TS in R0 resection. R0 resection is considered a significant predictor of improved survival as patients with $\mathrm{R} 0$ resection have shown a higher survival rate than those with incomplete resection (R1/R2) $(24,25)$. To determine whether the VATS approach can achieve R0 resection, additional RCTs are needed.

Although VATS thymectomies are being increasingly performed and have several advantages, indications for them are still controversial. Hida et al. (26) suggested that mediastinal tumors larger than $7 \mathrm{~cm}$ might not be suitable for VATS. More data are needed to define the indications for VATS.

\section{Limitations}

Our meta-analysis has some limitations. First, it included only nonrandomized, retrospective studies. Second, considering the advances in VATS technology, we excluded an article published in 2008 that reported eight cases (27). This may have resulted in selection bias. Third, in our clinical practice, we found that young female patients preferred VATS over TS, which again would have increased the risk of selection bias. Fourth, our study did not focus on the potential differences between partial and total thymectomy, although some studies have suggested the efficacy of partial thymectomy for early-stage thymoma (28-30). Fifth, most of the included studies were conducted in China and Japan, which also would have been a source of selection bias due to differences in the selection of approaches for early-stage thymoma between different geographic regions (31). Lastly, we still lack adequate longterm outcome data to analyze the survival rates between VATS and TS approaches for early-stage thymomas.

\section{Conclusions}

For early-stage thymoma patients, VATS thymectomy seems to provide many advantages to be considered as a legitimate alternative to TS; however, when performing VATS, surgeons should pay special attention to ensure that $\mathrm{R} 0$ resection is achieved.

\section{Acknowledgments}

The authors thank AsiaEdit for the help in language editing. Funding: None. 


\section{Footnote}

Conflicts of Interest: All authors have completed the ICMJE uniform disclosure form (available at http://dx.doi. org/10.21037/gs.2020.03.10). The authors have no conflicts of interest to declare.

Ethical Statement: The authors are accountable for all aspects of the work in ensuring that questions related to the accuracy or integrity of any part of the work are appropriately investigated and resolved.

Open Access Statement: This is an Open Access article distributed in accordance with the Creative Commons Attribution-NonCommercial-NoDerivs 4.0 International License (CC BY-NC-ND 4.0), which permits the noncommercial replication and distribution of the article with the strict proviso that no changes or edits are made and the original work is properly cited (including links to both the formal publication through the relevant DOI and the license). See: https://creativecommons.org/licenses/by-nc-nd/4.0/.

\section{References}

1. Mineo TC, Sellitri F, Ambrogi V. Left-sided video-assisted thoracic surgery thymectomy. Video-assist Thorac Surg 2017;2:32.

2. Raza A, Woo E. Video-assisted thoracoscopic surgery versus sternotomy in thymectomy for thymoma and myasthenia gravis. Ann Cardiothorac Surg 2016:5:33.

3. Seo J, Doerr F, Heldwein M, et al. Is Video-Assisted Thoracoscopic Surgery (VATS) a Worthy Alternative to Median Sternotomy in Resecting Stage I and II Thymoma? Thorac Cardiovasc Surg 2017:65:OP26.

4. Li J F, Hui B G, Li X, et al. Video-assisted thoracic surgery for thymoma: long-term follow-up results and prognostic factors-single-center experience of 150 cases. J Thorac Dis 2018:10:291.

5. Moher D, Liberati A, Tetzlaff J, et al. Preferred reporting items for systematic reviews and meta-analyses: the PRISMA statement. Ann Intern Med 2009:151:264-9.

6. Slim K, Nini E, Forestier D, et al. Methodological index for non-randomized studies (MINORS): development and validation of a new instrument. ANZ J Surg 2003:73:712-6.

7. Agatsuma H, Yoshida K, Yoshino I, et al. Videoassisted thoracic surgery thymectomy versus sternotomy thymectomy in patients with thymoma. Ann Thorac Surg
2017:104:1047-53.

8. Chao YK, Liu YH, Hsieh MJ, et al. Long-term outcomes after thoracoscopic resection of stage I and II thymoma: a propensity-matched study. Ann Surg Oncol 2015:22:1371-6.

9. Gu ZT, Mao T, Chen WH, et al. Comparison of videoassisted thoracoscopic surgery and median sternotomy approaches for thymic tumor resections at a single institution. Surg Laparosc Endosc Percutan Tech 2015:25:47-51.

10. Liu TJ, Lin MW, Hsieh MS, et al. Video-assisted thoracoscopic surgical thymectomy to treat early thymoma: a comparison with the conventional transsternal approach. Ann Surg Oncol 2014:21:322-8.

11. Ye B, Tantai JC, Ge XX, et al. Surgical techniques for early-stage thymoma: video-assisted thoracoscopic thymectomy versus transsternal thymectomy. J Thorac Cardiovasc Surg 2014:147:1599-603.

12. Yuan ZY, Cheng GY, Sun KL, et al. Comparative study of video-assisted thoracic surgery versus open thymectomy for thymoma in one single center. J Thorac Dis 2014:6:726.

13. Fadayomi AB, Iniguez CEB, Chowdhury R, et al. Propensity Score Adjusted Comparison of Minimally Invasive versus Open Thymectomy in the Management of Early Stage Thymoma. Thorac Cardiovasc Surg 2018:66:352-8.

14. Odaka M, Akiba T, Yabe M, et al. Unilateral thoracoscopic subtotal thymectomy for the treatment of stage I and II thymoma. Eur J Cardiothorac Surg 2010:37:824-6.

15. Pennathur A, Qureshi I, Schuchert MJ, et al. Comparison of surgical techniques for early-stage thymoma: feasibility of minimally invasive thymectomy and comparison with open resection. J Thorac Cardiovasc Surg 2011:141:694-701.

16. Kimura T, Inoue $M$, Kadota $Y$, et al. The oncological feasibility and limitations of video-assisted thoracoscopic thymectomy for early-stage thymomas. Eur J Cardiothorac Surg 2013:44:e214-8.

17. Sakamaki Y, Oda T, Kanazawa G, et al. Intermediate-term oncologic outcomes after video-assisted thoracoscopic thymectomy for early-stage thymoma. J Thorac Cardiovasc Surg 2014:148:1230-7.e1.

18. Toker A, Erus S, Ozkan B, et al. Does a relationship exist between the number of thoracoscopic thymectomies performed and the learning curve for thoracoscopic resection of thymoma in patients with myasthenia gravis? Interact Cardiovasc Thorac Surg 2011:12:152-5. 
19. Lucchi M, Davini F, Ricciardi R, et al. Management of pleural recurrence after curative resection of thymoma. J Thorac Cardiovasc Surg 2009:137:1185-9.

20. Gummert JF, Barten MJ, Hans C, et al. Mediastinitis and cardiac surgery-an updated risk factor analysis in 10,373 consecutive adult patients. Thorac Cardiovasc Surg 2002:50:87-91.

21. Losanoff JE, Richman BW, Jones JW. Disruption and infection of median sternotomy: a comprehensive review. Eur J Cardiothorac Surg 2002:21:831-9.

22. Friedant AJ, Handorf EA, Su S, et al. Minimally invasive versus open thymectomy for thymic malignancies: systematic review and meta-analysis. J Thorac Oncol 2016:11:30-8.

23. Higgins JP, Green S. Cochrane handbook for systematic reviews of interventions. John Wiley \& Sons, 2011.

24. Chen KN, Fu H, Gu Z, et al. P01: Long-term survival after surgical treatment of thymic carcinoma. J Thorac Dis 2015;7:AB070.

25. Infante MV, Voulaz E, Passera E, et al. Surgical management of thymoma and thymic carcinoma: results in 158 patients. Interact Cardiovasc Thorac Surg 2014:19:S25-6.

Cite this article as: Rusidanmu A, Feng M, Xu J, Wang L, $\mathrm{He} \mathrm{C}, \mathrm{Hu}$ J. Trans-sternotomy versus video-assisted thoracic surgery for early-stage thymoma patients: a meta-analysis. Gland Surg 2020;9(2):342-351. doi: 10.21037/gs.2020.03.10
26. Hida Y, Muto J, Kaga K, et al. Indication of video-assisted thoracic surgery for mediastinal mass lesions. Kyobu geka. Kyobu Geka 2012:65:934-8.

27. Cheng YJ. Videothoracoscopic resection of encapsulated thymic carcinoma: retrospective comparison of the results between thoracoscopy and open methods. Ann Surg Oncol 2008:15:2235-8.

28. Yano M, Fujii Y, Yoshida J, et al. A Phase II study of partial and subtotal thymectomy for thymoma (JART02). World J Surg 2017:41:2033-8.

29. Nakagawa K, Asamura H, Sakurai H, et al. Does the mode of surgical resection affect the prognosis/recurrence in patients with thymoma? J Surg Oncol 2014:109:179-83.

30. Voulaz E, Veronesi G, Infante M, et al. Radical thymectomy versus conservative thymomectomy in the surgical treatment of thymic malignancies. J Thorac Dis 2018:10:4127.

31. Fang W, Yao X, Antonicelli A, et al. Comparison of surgical approach and extent of resection for stage $i$ and ii thymic tumours in europe, north america and asia: an itmig retrospective database geographic analysis. Interact Cardiovasc Thorac Surg 2016:23:i1. 